\title{
IMPACTO DE UN PROGRAMA DE REHABILITACIÓN PULMONAR SOBRE VARIABLES CLINICAS, PSICOSOCIALES Y DE LA CAPACIDAD DE EJERCICIO EN PACIENTES CON ENFERMEDAD PULMONAR INTERSTICIAL A 2640 METROS SOBRE EL NIVEL DEL MAR Luisa Fernanda Güell ${ }^{1,2}$, Carlos Matiz $C^{1,2}$, Rafael Acero ${ }^{1,2}$

1. Fundación Santa Fe de Bogotá, 2. Universidad de los Andes.

\section{Introducción}

La enfermedad pulmonar intersticial incluye una serie de enfermedades poco frecuentes, aproximadamente 200, que comparten un cuadro clínico, radiológico, funcional y patológico con unos patrones determinados, que nos permiten identificar las diferentes etiologías y pronósticos. Existe una creciente evidencia que la rehabilitación pulmonar es una intervención requerida en pacientes con enfermedad intersticial, para disminuir el impacto de la enfermedad con respecto a los síntomas (disnea), la capacidad funcional y las variables psicosociales, independientemente de su etiología y severidad. A 2640 metros sobre el nivel del mar no hay estudios que evidencien dicho beneficio.

\section{Objetivos}

Evaluar el impacto de un Programa de Rehabilitación Pulmonar (PRP) sobre variables clínicas, psicosociales y de la capacidad de ejercicio en pacientes con enfermedad pulmonar intersticial a 2640 metros sobre el nivel del mar.

\section{Materiales y métodos}

Estudio descriptivo de pacientes con diagnóstico clínico, radiológico o patológico que se encontraban en tratamiento ambulatorio en un Hospital de alta complejidad, a los cuales se les realizó un Programa de Rehabilitación Pulmonar ambulatorio de 8 semanas, tres veces por semana, el cual incluía entrenamiento físico y educación. Se evaluaron variables clínicas, psicosociales y capacidad de ejercicio al inicio y al final del programa. Se siguieron durante un año para número de reingresos hospitalarios.

\section{Resultados}

Ingresaron al Programa de Rehabilitación Pulmonar (PRP) 45 pacientes con diagnóstico de enfermedad intersticial. Treinta y un pacientes realizaron en forma completa el programa, 20 hombres y 17 mujeres con edad promedio de $68.4+/-12.4$ años. En la Tabla 1 se describen las características clínicas de la población. Diez y ocho $(58,1 \%)$ de los pacientes tenían diagnóstico de fibrosis pulmonar idiopática; $5(16,1 \%)$ fibrosis secundaria a enfermedades del Colágeno; 6 (19,4\%) secundarias a medicamentos y $2(6,5 \%)$ granulomatosas. En la Tabla 2 se observan los cambios en las variables clínicas postrehabilitación pulmonar con mejoría significativa en la disnea y el número de hospitalizaciones. En la Tabla 3 se observan los cambios post-rehabilitación en la capacidad de ejercicio, con mejoría significativa en la distancia caminada, los mets alcanzados y la disnea de esfuerzo. En las variables psicosociales se observó mejoría significativa en depresión (Beck II, $p=0.005$ ) y en calidad de vida (SGRQ) (Figura 1), pero no se observó mejoría en los niveles de ansiedad (STAI).

\begin{tabular}{|c|c|c|c|}
\hline Variables & $\begin{array}{c}n=31 \\
\text { media (SD) }\end{array}$ & Variables & $\begin{array}{c}n=31 \\
\text { media (SD) }\end{array}$ \\
\hline Disnea mMRC & $2,2(0,9)$ & $\%$ CPT del predicho & $68,6(10,8)$ \\
\hline Índice de CHARLSON & $3,2(1,3)$ & \%Volumen residual (VR) & $66,6(15,9)$ \\
\hline Indice de masa corporal & $25,1(4,5)$ & VR/CPT & $45,3(17,5)$ \\
\hline $\mathrm{VEF}_{1}(\mathrm{ml})$ & $1,89(0,4)$ & DLCO absoluto $(n=22)$ & $47,09(14,2)$ \\
\hline$\% \mathrm{VEF}_{1}$ del predicho & $74,8(23,5)$ & DLCO ajustada $(n=22)$ & $46,5(15,7)$ \\
\hline Relación VEF 1 /CV & $84,6(8,1)$ & Horas de oxigeno & $15,4(8,4)$ \\
\hline Capacidad vital forzada & $71,2(17,9)$ & $\mathrm{FiO}_{2}$ para el ejercicio & $0,45(0,23)$ \\
\hline Capacidad pulmonar total & $3,8(0,6)$ & Saturación de $\mathrm{O}_{2}$ en reposo & $88,6(5,9)$ \\
\hline
\end{tabular}

Tabla 2. Cambios post- PRP en las variables clínicas

$\begin{array}{llll}\text { Variables Clínicas } & \text { Pre RHP }(n=31) & \text { Post RHP }(n=31) & \mathrm{p} \\ \text { Disnea (mMRC) } & 2,26(0,9) & 1,52(1,2) & \mathrm{p}<0,0002^{*} \\ \text { Índice de Masa Corporal } & 25,1(4,5) & 24,85(4,6) & \mathrm{P}=0,48 \\ \text { Numero de hospitalizaciones } & * * 0,9(0,9) & * * * 0,43(0,7) & \mathrm{P}=0,05^{*} \\ \text { Días de Hospitalización } & * * 12,32(21 ; 6) & * * * 4,0(8,9) & \mathrm{P}=0,06\end{array}$

Wilcoxon signed Rank test: Cambio significativo $p \leq 0,05$; ** un año previo al PRP *** Un año posterior al PRP

Tabla 3. Cambios post-PRP en las variables de capacidad de ejercicio

\begin{tabular}{llll} 
Capacidad ejercicio & Pre RHP $(n=31)$ & Post RHP $(n=31)$ & $p$ \\
\hline Distancia en metros (C6M) & $418,8(97,4)$ & $471,43(112,1)$ & $p=0,0004^{*}$ \\
Mets & $2,98(0,5)$ & $3,25(0,5)$ & $p=0,0004^{*}$ \\
Disnea máxima (Borg) & $3,1(2,4)$ & $2,0(1,5)$ & $p=0,0004^{*}$ \\
Fatiga máxima (Borg) & $3,1(2,6)$ & $2,3(1.7)$ & $p=0,07$
\end{tabular}

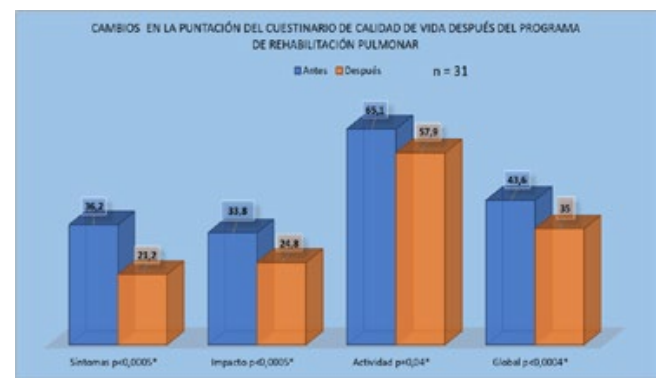

Gráfica 1. Cambios post-PRP en la calidad de vida (SGRQ) Conclusiones

En los pacientes con enfermedad pulmonar intersticial residentes a 2640 metros sobre el nivel del mar, la Rehabilitación Pulmonar como parte del tratamiento, permite mejoría significativa en variables clínicas, funcionales (caminata de seis minutos) y psicosociales (Inventario de Depresión de Beck II, Calidad de vida por SGRQ).

\section{Referencias}

1. Vainshelboim B, Oliveira J, Yehoshua L, Weiss I, Fox BD, Fruchter O, et al. Exercise TrainingBased Pulmonary Rehabilitation Program Is Clinically Beneficial for Idiopathic Pulmonary Fibrosis. Respiration; 88(5):378-88.

2. Swigris JJ, Brown KK, Make BJ, Wamboldt FS. Pulmonary rehabilitation in idiopathic pulmonary fibrosis: A call for continued investigation. Respir Med 102(12):1675-80.

3. Kozu R, Senjyu H, Jenkins SC, Mukae H, Sakamoto N, Kohno S. Differences in Response to Pulmonary Rehabilitation in Idiopathic Pulmonary Fibrosis and Chronic Obstructive Pulmonary Disease. Respiration 2011: 11];81(3):196-205.

4. Betancourt-Peña J, Hurtado-Gutiérrez H. Efectos de un programa de rehabilitación pulmonar en pacientes con enfermedad pulmonar Intersticial difusa. Fisioterapia 2015; 37(6):286-92. 